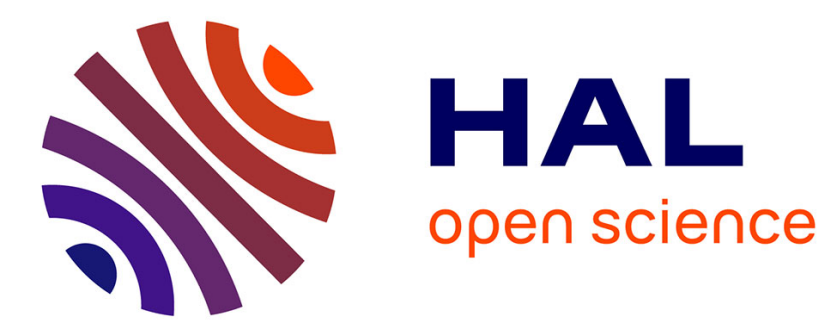

\title{
Control of the textural properties of nanocrystalline boehmite $(\gamma$-AlOOH $)$ regarding its peptization ability
}

Fouad Karouia, Malika Boualleg, Mathieu Digne, Pierre Alphonse

\section{To cite this version:}

Fouad Karouia, Malika Boualleg, Mathieu Digne, Pierre Alphonse. Control of the textural properties of nanocrystalline boehmite $(\gamma-\mathrm{AlOOH})$ regarding its peptization ability. Powder Technology, 2013, vol. 237, pp. 602-609. 10.1016/j.powtec.2012.12.054 . hal-01167383

\section{HAL Id: hal-01167383 \\ https://hal.science/hal-01167383}

Submitted on 24 Jun 2015

HAL is a multi-disciplinary open access archive for the deposit and dissemination of scientific research documents, whether they are published or not. The documents may come from teaching and research institutions in France or abroad, or from public or private research centers.
L'archive ouverte pluridisciplinaire HAL, est destinée au dépôt et à la diffusion de documents scientifiques de niveau recherche, publiés ou non, émanant des établissements d'enseignement et de recherche français ou étrangers, des laboratoires publics ou privés. 


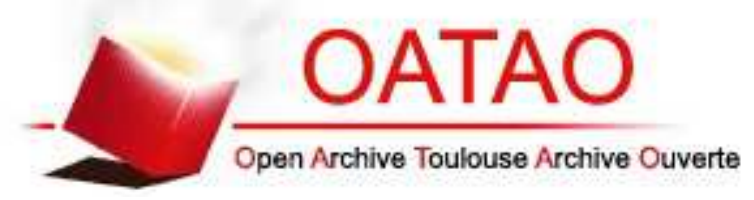

\section{Open Archive TOULOUSE Archive Ouverte (OATAO)}

OATAO is an open access repository that collects the work of Toulouse researchers and makes it freely available over the web where possible.

This is an author-deposited version published in : http://oatao.univ-toulouse.fr/ Eprints ID : 13997

To link to this article : doi: 10.1016/j.powtec.2012.12.054

URL : http://dx.doi.org/10.1016/i.powtec.2012.12.054

To cite this version : Karouia, Fouad and Boualleg, Malika and Digne, Mathieu and Alphonse, Pierre Control of the textural properties of nanocrystalline boehmite $(\gamma-\mathrm{AlOOH})$ regarding its peptization ability. (2013) Powder Technology, vol. 237. pp. 602-609. ISSN 0032-5910

Any correspondance concerning this service should be sent to the repository administrator: staff-oatao@ listes-diff.inp-toulouse.fr 


\title{
Control of the textural properties of nanocrystalline boehmite $(\gamma-\mathrm{AlOOH})$ regarding its peptization ability
}

\author{
Fouad Karouia ${ }^{\text {a,b }}$, Malika Boualleg ${ }^{\text {a,* }}$, Mathieu Digne ${ }^{\text {a }}$, Pierre Alphonse ${ }^{\text {b }}$ \\ a IFP Energies nouvelles, Rond-point de l'échangeur de Solaize, BP 3, 69360 Solaize, France \\ b Université de Toulouse, CIRIMAT UPS-CNRS, 118 route de Narbonne, 31062 Toulouse Cedex 9, France
}

Keywords:

Boehmite

Sol-gel

Precipitation

Peptization

Neutralization

Kneading-extrusion

\begin{abstract}
A B S T R A C T
Kneading-extrusion is a key shaping process, especially used to prepare extrudates for catalytic applications. It usually involves several successive steps, mainly used to control textural properties such as pore diameter and pore volume of $\gamma$-alumina catalyst supports. The ability to successfully tune these properties is highly dependent on the boehmite precursor features. This work aims at understanding the influence of the peptization and neutralization steps on the textural properties of boehmite exhibiting similar crystallite size and shape but different peptization abilities. Three kinds of boehmite (low, medium and high peptization ability) obtained either by precipitation or by alkoxide hydrolysis were subjected to a peptization and neutralization steps in order to simulate the full shaping process of kneading extrusion. After peptization and neutralization, no significant changes were found in crystallite shape and size, but only in their spatial arrangement. For the boehmite exhibiting the lowest peptization ability, the particle packing seems to be frozen and no modifications result from peptization or neutralization. However for boehmite with the medium peptization ability, obtained by precipitation from the same precursors as the low peptizable sample, the particles were initially poorly organised and their spatial packing was very affected by the peptization and neutralization phases, giving a narrower pore size distribution than the synthesized powder. Though the third boehmite, obtained by alkoxide hydrolysis, was fully peptizable, this does not allow tailoring the textural properties, whatever the phase of the shaping process. Finally the partially peptizable boehmite seems to be the most versatile starting material for the control of the textural properties.
\end{abstract}

\section{Introduction}

Nanocrystalline boehmites are of primary interest because of their technical importance in the preparation of controlled porosity catalysts with enhanced textural and mechanical properties [1-4]. The basic properties of the catalyst component are, of course, the main determinant, but texture and stability of the support can be strongly influenced by the preparation method [5-7]. Boehmite $(\mathrm{AlOOH})$ is mainly obtained either by hydrolysis of Al-alkoxides or by precipitation of aluminium salts. Matijevic et al. [8-10] and Panias et al. [11] have shown that the properties of boehmite, such as particle size, shape and structure, depend on both the nature of aluminium precursors and the synthesis conditions. As-synthesized boehmites are agglomerates of aggregated crystallites (primary particles). The aggregation is considered as a non reversible phenomenon resulting from a chemical bonding of the crystallites, whereas agglomeration is a reversible

\footnotetext{
* Corresponding author. Tel.: +334377020 21; fax: +33437702066.

E-mail addresses: fouad.karouia@ifpen.fr (F. Karouia), malika.boualleg@ifpen.fr (M. Boualleg), mathieu.digne@ifpen.fr (M. Digne), pierre.alphonse@chimie.ups-tlse.fr
} (P. Alphonse). phenomenon involving weaker bonds such as attractive electrostatic forces or Van der Waals bonds [4]. The homogeneous stable suspensions of the material are achieved by reducing the agglomerates to a sufficiently small size such that they remain suspended as a colloid [12]. This process known as peptization, or deagglomeration, is usually achieved by adsorption of protons or hydroxyl ions on the surface of the particles causing electrostatic repulsion. Petrovic et al. [13] have shown that textural properties, such as specific surface area, depend on the type of acid used for the peptization during the synthesis step, which means that the deagglomeration state depends on the used acid. Morgado et al. [14] studied the boehmite particles' behaviour regarding peptization for samples synthesized at high $\left(85^{\circ} \mathrm{C}\right)$ and room $\left(25^{\circ} \mathrm{C}\right.$ ) temperature and for various $\mathrm{OH} / \mathrm{Al}$ ratios. They found, for example, that hydrolysis at $85{ }^{\circ} \mathrm{C}$ does not favour the formation of peptizable boehmites. Boehmite gels can be peptized, by means of various acids, to make metastable sols or homogeneous pastes $[3,15,16]$. In the latter case this step is widely used to prepare shaped catalyst support. One of the most used techniques to shape support catalyst is kneadingextrusion where a concentrated suspension or paste is passed through a profiled die that determines the final shape of the support. The kneading-extrusion process includes (i) a knead of the powder with liquid (usually water, with organic and mineral additives), (ii) extrusion 
of the paste and (iii) drying of the shaped support [17]. Extruding equipment are screw extruders or press extruders. Press extruders are principally used for pastes with low moisture and high compaction. The homogeneity of the paste is an essential parameter in order to insure that the quality of the product is kept constant. To improve this homogeneity, additives can be used, especially acids or organic additives, to minimise interparticle crosslinking [18]. Moreover the quantity of acid permits to control the agglomerates size thus the pore size distribution can be tailored [19]. These additives are also used to tune the paste viscosity because a too viscous paste will block the extruder whereas a product that lacks viscosity will give extrudates without mechanical resistance [19]. However, the influence of a full shaping process on textural and structural properties as a function of boehmite properties has not been studied in detail in literature. The present paper deals with a shaping process by kneading-extrusion which is commonly used to produce catalyst support. The process reported here includes an acidic knead of the powder with diluted nitric acid in order to reduce and homogenize the agglomerates size. A basic knead was then carried out by addition of ammonia solution to the paste to increase the mean pore diameter [20]. The obtained paste was then extruded, dried and calcined to obtain the catalyst support.

In this work we design a method to estimate the peptization ability of a boehmite powder. Thus we can evaluate the influence of the peptization ability of boehmite sample on the textural properties change. Three samples with very different peptization ability but with similar shape and size of crystallites were synthesized. To study the effect of the acidic and basic knead, we carried out these two steps separately. Thus we can compare the texture of as synthesized, peptized, neutralized and fully shaped boehmite. After each step, the product was characterised by X-ray diffraction (XRD), $\mathrm{N}_{2}$ adsorption-desorption isotherm (in order to determine the specific surface area using the BET method and the pore size distribution calculated by DFT) and transmission electron microscopy (TEM).

\section{Experimental}

\subsection{Boehmite preparation}

Precipitated boehmites were synthesized by neutralizing an aluminium sulphate solution with a sodium aluminate solution under stirring. The first boehmite was obtained at $60{ }^{\circ} \mathrm{C}$ and $\mathrm{pH} 9.7$ by simultaneous dosing as reported by Morgado et al. [14]. The gel obtained was then washed five times with hot water in order to minimise impurities content $\left(\mathrm{Na}^{+}\right.$and $\left.\mathrm{SO}_{4}^{2-}\right)$. After drying at $120{ }^{\circ} \mathrm{C}$ overnight, the obtained solid was ground. This dried boehmite is denoted LP which stands for low peptization ability. The second boehmite is an industrial product, provided by Axens (www.axens.net), prepared by precipitation from the same precursors as the LP boehmite and is referred to as MP (medium peptization ability). Compared to LP boehmite, the industrial conditions lead to a more peptizable product.

In order to obtain a highly peptizable boehmite (HP), a material was synthesized by hydrolysis of an aluminium alkoxide, according to the process originally reported by Yoldas [21]. A large excess $\left(\mathrm{H}_{2} \mathrm{O} / \mathrm{Al} \approx 100\right)$ of hot $\left(85^{\circ} \mathrm{C}\right)$ distilled water was quickly poured in aluminium tri-sec-butoxide, $\mathrm{Al}\left(\mathrm{OC}_{4} \mathrm{H}_{9}\right)_{3}$ under stirring (350 rpm), which was maintained for $15 \mathrm{~min}$. The obtained sol is dried at $80{ }^{\circ} \mathrm{C}$ and then grounded. This boehmite is referenced as HP.

In order to understand the impact of each step on the evolution of textural properties, the full shaping process by kneading-extrusion was decomposed in elementary steps defined as following:

- First a peptization step with nitric acid was done using an Ultraturax ${ }^{\circledR}$ disperser,

- Second a neutralisation step was carried out by adding ammonia also in the Ultraturax ${ }^{\circledR}$ disperser,
- Finally, a whole shaping process by keanding-extrusion was done using a Brabender ${ }^{\circledR}$ mixer for the knead of the paste and press extruder for the extrusion process,

- For comparison, the MP sample was analysed after kneading using the Brabender ${ }^{\circledR}$ mixer without undergoing the extrusion process.

For all boehmites, the peptization step was done by adding $\mathrm{ca} .0 .025$ mole of nitric acid ( $\mathrm{HNO}_{3} 68 \%$ GPR RECTAPUR) per mole of aluminum. The desired amount of acid was added to distilled water and mixed for $2 \mathrm{~min}$. The boehmite was weighed and added to the water-acid mix (water/powder wt $\approx 7$ ) under very vigorous stirring using an Ultraturax ${ }^{\circledR}$ disperser at $13,000 \mathrm{rpm}$ until obtaining an homogeneous suspensions (about $5 \mathrm{~min}$ ). The sample name with the extension -P refers to the peptized sample. For example the peptized HP sample is denoted HP-P. Then neutralization step was carried out using ammonia (28\% solution) at $40 \mathrm{wt} . \%$ according to the mass of acid used for peptization. The extension $-\mathrm{N}$ is added to refer to neutralized samples. Excepting extrudates all other samples were peptized and neutralised using an Ultraturax ${ }^{\circledR}$ disperser.

For extrudates, the powder knead was done in a Brabender ${ }^{\circledR}$ mixer (a tank of $80 \mathrm{~cm}^{3}$ ) working at $33 \mathrm{rpm}$. First an acid kneading step was done using nitric acid $(\mathrm{H} / \mathrm{Al} \approx 0.025)$, then a basic knead was done using ammonia. The knead time takes about $30 \mathrm{~min}$ and the solid on water ratio was about 0.5 . The obtained paste was then extruded in a press extruder using a $2 \mathrm{~mm}$ cylindrical die. The obtained samples are dried and referenced as extrudates. Unless otherwise stated, all samples are dried overnight in an oven at $80{ }^{\circ} \mathrm{C}$.

\subsection{Peptization index (PI)}

The peptization is a qualitative concept that qualifies the powder ability to be dispersed in a liquid media. Several methods have been proposed to quantify this ability (see for instance, [22]). In the present study, a peptization index was determined in an acidic media as follow: boehmite suspensions (10 wt.\%) were centrifuged $10 \mathrm{~min}$ at $3000 \mathrm{rpm}$. The sediments are considered as the non peptizable part (NP). The particles remaining in suspension are considered as the peptizable part $(\mathrm{P})$. These two parts were then dried overnight in an oven at $80^{\circ} \mathrm{C}$. Finally the peptization index (PI) is defined as the ratio of the mass of the peptizable part on the sum of masses of peptizable and non peptizable parts:

$P I=\frac{m_{P}}{m_{P}+m_{N P}}$

\subsection{Characterization}

The crystal phase identification was done by powder X-ray diffraction (PXRD). Data were collected on a PANalytical X'Pert Pro $\theta-\theta$ diffractometer in Bragg-Brentano geometry, using filtered $\mathrm{Cu} \mathrm{K} \alpha$ radiation and a graphite secondary-beam monochromator. Diffraction intensities were measured, at room temperature, by scanning from 2 to $72^{\circ}$ with a step size of $0.05^{\circ}(2 \theta)$. Crystallite sizes were determined by Scherrer's equation. In order to accurately determine the shape and size of crystallites, experimental X-ray patterns were compared to simulated morphology-dependent patterns diffraction using the Debye formula according to the method described elsewhere [23]. Briefly, this formula allows to calculate the diffraction pattern of a finite size atomic structure. The intensity $\mathrm{I}(\theta)$ of electron units scattered at an angle $2 \theta$ is expressed by the following formula.

$I(\theta)=\sum_{i} \sum_{j} f_{i} f_{j} \frac{\sin \left(\frac{4 \pi r_{i j} \sin (\theta)}{\lambda}\right)}{\frac{4 \pi r_{i j} \sin (\theta)}{\lambda}}$

where $f_{i}$ is the atomic scattering factor of the atom $i, r_{i j}$ is the distance between the atoms $i$ and $j$, and $\lambda$ is the wavelength of the incident 
$\mathrm{X}$-rays. The numerical calculation has been performed as implemented in the SIMVAX program [24]. Each boehmite particle can be fully described by four distances $\left(D_{a}, D_{b}, D_{c}\right.$, and $\left.D_{d}\right)$ corresponding to the edge lengths of the particles and defining the exposed surface area of each face (Fig. 1).

The agreement between the experimental and calculated patterns is quantified using the weighted $R_{\mathrm{wp}}$ factor defined in Eq. (3) and visual comparison of the relative intensities.

$R_{w p}=\left(\frac{\sum_{i} w_{i}\left[c I^{\text {sim }}\left(\theta_{i}\right)-I^{\exp }\left(\theta_{i}\right)+I^{\text {bckg }}\left(\theta_{i}\right)\right]^{2}}{\sum_{i} w_{i}\left[I^{\exp }\left(\theta_{i}\right)\right]^{2}}\right)^{1 / 2}$

The best agreement corresponds to the lowest $R_{w p}$.

Transmission electron microscopy (TEM) observations were performed on a JEOL2100F-FEG (Field emission gun) apparatus. A small amount of powder was dispersed in water using an ultrasound bath. Then a droplet was deposited on a carbon-coated grid and allowed to dry under UV lamp.

The nitrogen adsorption-desorption isotherms were collected at $77 \mathrm{~K}$ using an adsorption analyser Micromeritics ASAP 2420. Samples were dried beforehand under vacuum for $6 \mathrm{~h}$ at $110{ }^{\circ} \mathrm{C}$. From $\mathrm{N}_{2}$ isotherm, specific surface area (SSA) was determined by the BET method [25] and pore size distribution (PSD) was calculated by the NLDFT (nonlocal density functional theory) [26] model assuming a cylindrical pore structure (software Autosorb 1 from Quantachrome Instruments). The quality of the agreement between experimental and calculated isotherms was evaluated by the profile factor $R_{p}$ :

$R_{p}=100 \sum\left|y_{i o}-y_{i c}\right| / \sum y_{i o}$

The mean pore diameter (D) corresponds to the maximum of the pore size distribution. When the PSD is not monomodal, we report two $\mathrm{D}$ values corresponding to the two observed local maxima.

Pore volume $\left(\mathrm{P}_{\mathrm{V}}\right)$ was calculated from the adsorbed volume of gas, $\mathrm{V}_{\mathrm{a}}$ at the higher relative pressure $\left(\mathrm{P} / \mathrm{P}_{0} \approx 0.99\right)$ by:

$P_{V}=\frac{N_{2} \text { gas density }}{N_{2} \text { liq density }} * V_{a}=0.00155 \times V_{a}$

The relative errors associated with adsorption-desorption analyses were estimated to be $5 \%$ for the specific surface area (SSA), pore volume and $20 \%$ for the pore diameter (D).

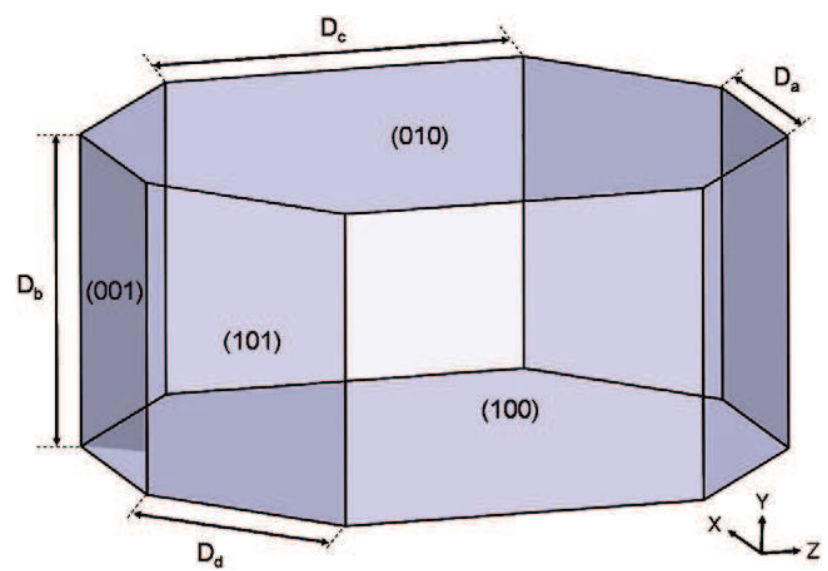

Fig. 1. Morphological model of a boehmite nanoparticles. $D_{a}, D_{b}, D_{c}$, and $D_{d}$ are the lengths of the four particle edges.

\section{Results}

\subsection{XRD of boehmite samples}

\subsubsection{As synthesized samples}

For all samples, the only detected phase on XRD patterns (Fig. 2) was boehmite (ICDD 21-1307 card). Moreover, the ${ }^{27}$ Al MAS NMR spectra (see supporting information) did not show any presence of tetrahedral aluminium $\left(\mathrm{Al}^{\mathrm{IV}}\right)$ but only octahedral aluminium $\left(\mathrm{Al}^{\mathrm{VI}}\right)$ which means that all samples are crystalline boehmite without amorphous boehmite.

The calculated crystallites size, in the (020) direction, is close to $3 \mathrm{~nm}$ for all samples (Table 2). Moreover, this size, within the experimental error, is in good agreement with the size estimated from XRD simulations. From XRD simulation we also see that the main faces are basal (010) and lateral (101) faces. This indicates that crystallites are diamond like shaped (Table 1).

The specific surface areas (SSA) are about $350 \mathrm{~m}^{2} / \mathrm{g}$ for the MP and HP boehmites but only $273 \mathrm{~m}^{2} / \mathrm{g}$ for the LP (Table 2). Although the two precipitated boehmite have a similar shape and size of crystallites (Table 1), the SSA is lower for LP than for MP. This is could be related to the high aggregation state of this sample [21].

The peptization index shows that the different synthesis conditions give three kinds of boehmites with peptization ability ranging from 0 to $100 \%$. The boehmite prepared by alkoxide hydrolysis (HP), can be considered as a fully peptizable $(\mathrm{PI} \approx 0.99)$, MP is partially peptizable $(\mathrm{PI} \approx 0.7)$, whereas $\mathrm{LP}$ is not peptizable $(\mathrm{PI} \approx 0)$. MP and LP samples, even if they are precipitated from the same precursors with similar content of impurities (Table 2), have different peptization ability. The pore volume of as synthesized boehmites vary from 0.35 to $0.76 \mathrm{~cm}^{3} / \mathrm{g}$ which is a good indication of the different state of aggregation between them. HP boehmite presents the more compact organisation of crystallites and thus the smaller mean pore diameter centred at $5.1 \mathrm{~nm}$. MP boehmite has a mean pore diameter centred at $9.5 \mathrm{~nm}$ and, for LP, the mean pore diameter is around $7.5 \mathrm{~nm}$.

\subsubsection{Mixed samples}

After the shaping process, either by the full kneading-extrusion or by the successive steps processes, similar trends were observed for the XRD patterns of the three boehmites, so herein we will present only the relevant data. For instance, X-ray diffraction patterns of typical shaped samples are presented in Fig. 3. All peaks correspond to boehmite phase according to ICDD 21-1307 card. In Fig. 3 it can be seen that there is no shift of peaks or significant modification of intensities after peptization, neutralization or kneading-extrusion. The thickness $\varepsilon_{020}$, along the $\mathrm{b}$

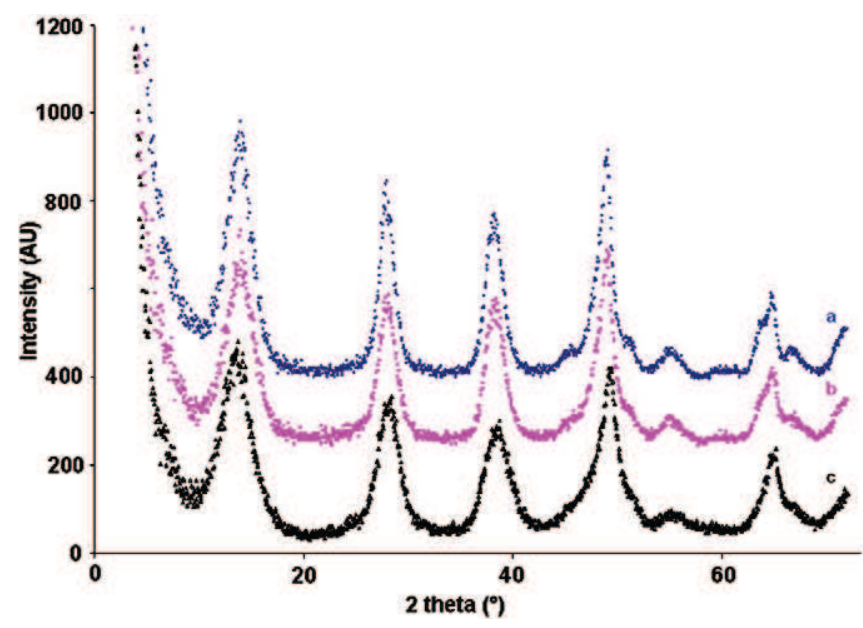

Fig. 2. XRD of as synthesized boehmite samples (a) LP, (b) MP and (c) HP. 
Table 1

Size and exposed face area for as synthesized boehmite samples.

\begin{tabular}{|c|c|c|c|c|c|c|c|c|c|}
\hline \multirow[t]{2}{*}{ Sample } & \multicolumn{4}{|c|}{ Particle size $(\mathrm{nm})$} & \multicolumn{4}{|c|}{ Exposed face area (\%) } & \multirow[t]{2}{*}{$\mathrm{V}\left(\mathrm{nm}^{3}\right)$} \\
\hline & $\mathrm{D}_{\mathrm{a}}$ & $\mathrm{D}_{\mathrm{b}}$ & $\mathrm{D}_{\mathrm{c}}$ & $\mathrm{D}_{\mathrm{d}}$ & $\mathrm{A}_{100}$ & $A_{010}$ & $A_{001}$ & $\mathrm{~A}_{101}$ & \\
\hline LP & 1.5 & 3.6 & 0.5 & 2.3 & 5 & 35 & 15 & 45 & 45 \\
\hline MP & 1.6 & 2.4 & 0.8 & 2.8 & 5 & 50 & 10 & 35 & 45 \\
\hline HP & 0.5 & 2.4 & 0.5 & 2.5 & 5 & 40 & 5 & 50 & 23 \\
\hline
\end{tabular}

axis, calculated by the Scherrer's equation and given in Table 3, is similar whatever the applied step of the shaping process.

For the as synthesized HP boehmite, a peak at $2 \theta=3.5^{\circ}$ was observed, indicating a regular spacing (corresponding to $2.5 \mathrm{~nm}$ ) between particles (insert in Fig. 3). It can be noticed that this distance is approximately the same that the thickness of crystallites.

For MP and HP boehmites, specific surface areas remain the same as the original material, within the experimental error (Table 3). As SSA is primary related to the particle size, this is in agreement with $\mathrm{XRD}$ results showing that there is no significant changes in crystallites shape and size. However a small increase in specific surface area (about $+30 \mathrm{~m}^{2} / \mathrm{g}$ ) is observed for LP boehmite extrudates.

\subsection{TEM observation on boehmite samples}

The particle shape of non peptizable LP boehmite (Fig 3A) is similar to the shape observed for HP (Fig. 4A). The micrograph shows randomly interconnected fibrils, whose length cannot be readily determined $\quad \sim 50$ to $200 \mathrm{~nm}$ ). These fibrils are organized as bundles and single crystallites are not visible. After peptization and neutralisation fibrils were still present and no change was observed on particle shape (Fig. 4B).

TEM micrographs of HP boehmite give further evidence of the very small size of the crystallites. Fibers with lengths ranging between $c a$. 10 and $50 \mathrm{~nm}$ are the main kind of agglomerates but we cannot readily determine the shape and size of single crystallites (Fig. 5A). In contrast, the peptized sample (HP-P) shows no specific morphology. The crystallites are distributed in a homogeneous fine and porous matrix (Fig. 5B). After neutralisation, the sample HP-PN is agglomerated in less organised way than the as synthesized HP. Fibers (20-30 nm length) are still present but irregular aggregates are also observed (Fig. 5C).

MP boehmite (partially peptizable) shows features between non peptizable and fully peptizable boehmites: both fiber-like and irregular aggregates are observed on the TEM micrographs.

Finally, for all samples, single crystallites are never visible in TEM micrographs, thus an estimation of their size or shape is impossible.

\subsection{Effect of the elementary steps on the porosity of boehmite samples}

The nitrogen adsorption-desorption isotherms and the pore size distributions (PSD) are given in Fig. 6. We have seen above that peptization has no effect on the spatial arrangement of crystallites for LP boehmite. The isotherms of type II with an H3 hysteresis loop, according to the IUPAC classification, obtained for all samples derived from LP sample tend to confirm this result. This kind of

Table 2

Characteristics of as synthesized boehmites.

\begin{tabular}{lllllllll}
\hline Sample & $\begin{array}{l}\varepsilon_{020} \\
(\mathrm{~nm})\end{array}$ & $\begin{array}{l}\varepsilon^{\mathrm{cal}}{ }_{020} \\
(\mathrm{~nm})\end{array}$ & $\begin{array}{l}\text { SSA } \\
\left(\mathrm{m}^{2} / \mathrm{g}\right)\end{array}$ & $\mathrm{P}_{\mathrm{V}}(\mathrm{ml} / \mathrm{g})$ & $\mathrm{D}(\mathrm{nm})$ & $\mathrm{PI}$ & $\mathrm{Na}(\mathrm{ppm})$ & $\mathrm{S}(\mathrm{ppm})$ \\
\hline $\mathrm{LP}$ & 3.3 & 3.6 & 273 & 0.66 & 7.5 & 0.0 & 57 & 270 \\
MP & 2.6 & 2.4 & 337 & 0.76 & 9.5 & 0.7 & 108 & 570 \\
HP & 2.5 & 2.3 & 358 & 0.35 & 5.1 & 0.99 & $\varnothing$ & $\varnothing$ \\
\hline
\end{tabular}

$\varepsilon_{020}$ and $\varepsilon^{\mathrm{cal}}{ }_{020}( \pm 0.5 \mathrm{~nm})$ : boehmite crystallite size in the direction (020) estimated by the Scherrer's formula and obtained from the simulated pattern respectively, SSA: specific surface Area, $\mathrm{P}_{\mathrm{v}}$ : pore volume estimated at $\mathrm{P} / \mathrm{P}_{0} \approx 0.99$, $\mathrm{D}$ : mean pore diameter calculated by the NLDFT method, PI: peptization index, and $\varnothing$ means lower than the detection limit.

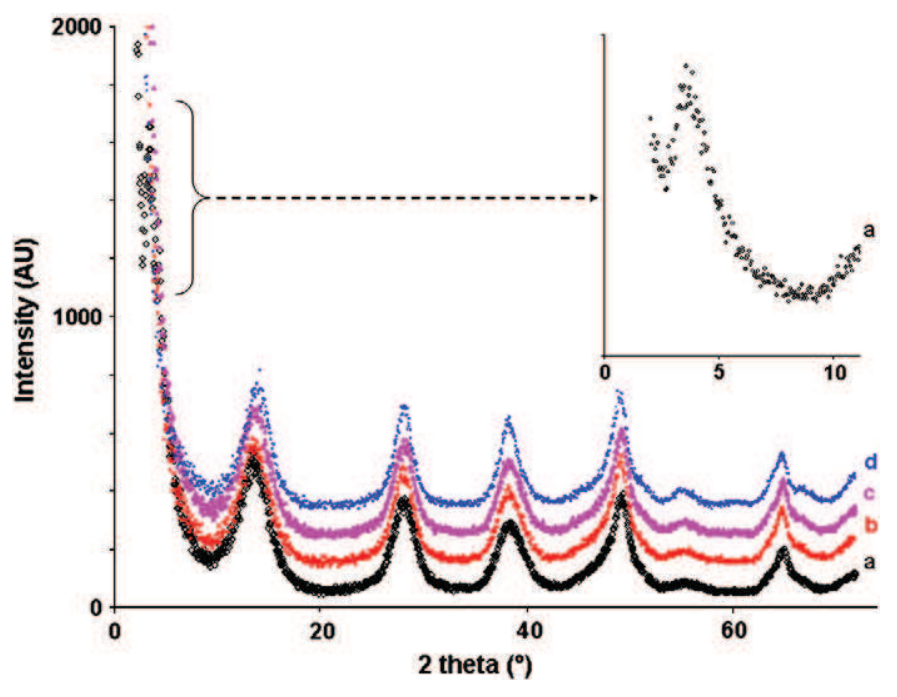

Fig. 3. XRD of HP as synthesized boehmite sample (a), peptized (b), neutralized (c) by the step shaping process and extrudates (d) derived from the HP sample.

hysteresis loop is observed for open slit-shaped pores with parallel walls. The same kind of isotherm was also obtained for the as precipitated MP boehmite. These boehmites have also a similar pore size distribution ranging from 5 to about $50 \mathrm{~nm}$ and centred at 7.5 and 9.5 for LP and MP respectively. No changes in isotherm type and PSD were observed after the shaping process for the samples derived from LP. However, the shaped samples derived from MP gave an isotherm of type IV with an $\mathrm{H} 2$ hysteresis loop. This hysteresis loop is observed for tubular pores or ink-bottle pores with short necks and wide sloping bodies. The PSD is narrower than the PSD of the as synthesized MP. The PSD of shaped sample ranged between 5 and $20 \mathrm{~nm}$ and are centred at 6.5, 6.5/9.5 and $9.5 \mathrm{~nm}$ for MP-P, MP-PN and extrudates respectively.

For HP boehmite, though TEM micrographs show that the spatial arrangement of crystallites was strongly affected by peptization and neutralization, no significant changes in the PSD of samples derived from HP were observed (except for HP-extrudates). All samples derived from HP gave an isotherm of type IV with an $\mathrm{H} 2$ hysteresis loop. The PSD of HP, HP-P and HP-PN ranged between $c a .3$ and $10 \mathrm{~nm}$ and are centred at $5.1 \mathrm{~nm}$. The sample shaped by kneadingextrusion has a larger pore size distribution that ranged between 3 and $15 \mathrm{~nm}$ and centred at 6.3 and $8.5 \mathrm{~nm}$.

Table 3

Summarizing of the boehmite samples characteristics.

\begin{tabular}{llllll}
\hline Sample & Treatment & $\varepsilon_{020}(\mathrm{~nm})$ & $\mathrm{SSA}\left(\mathrm{m}^{2} / \mathrm{g}\right)$ & $\mathrm{P}_{\mathrm{V}}(\mathrm{ml} / \mathrm{g})$ & $\mathrm{D}(\mathrm{nm})$ \\
\hline LP & $\varnothing$ & 3.3 & 273 & 0.66 & 7.5 \\
& $\mathrm{P}$ & 3.1 & 288 & 0.61 & 7.5 \\
& PN & 3.1 & 273 & 0.56 & 7.5 \\
$\mathrm{HP}$ & Extrudates & 3.1 & 308 & 0.53 & 7.5 \\
& $\varnothing$ & 2.6 & 337 & 0.76 & 9.5 \\
& P & 2.9 & 331 & 0.47 & 6.5 \\
$\mathrm{HP}$ & PN & 2.9 & 333 & 0.49 & $6.5 / 9.5$ \\
& Extrudates & 3.0 & 324 & 0.55 & 9.5 \\
& $\varnothing$ & 2.5 & 358 & 0.35 & 5.1 \\
& P & 2.4 & 360 & 0.31 & 5.1 \\
& PN & 2.5 & 364 & 0.32 & 5.1 \\
& Extrudates & 2.7 & 348 & 0.39 & $6.3 / 8.5$
\end{tabular}

Ø: no treatment, P: peptized samples, $\mathrm{N}$ : neutralized samples and extrudates refers to extruded samples. $\varepsilon( \pm 0.5 \mathrm{~nm})$ : boehmite particles size in the direction (020) estimated by the Scherrer's formula, SSA: specific surface Area, Pv: pore volume estimated at $\mathrm{P} / \mathrm{P}_{0} \approx 0.99$, D: mean pore diameter calculated by the DFT method on the adsorption branch. 
A

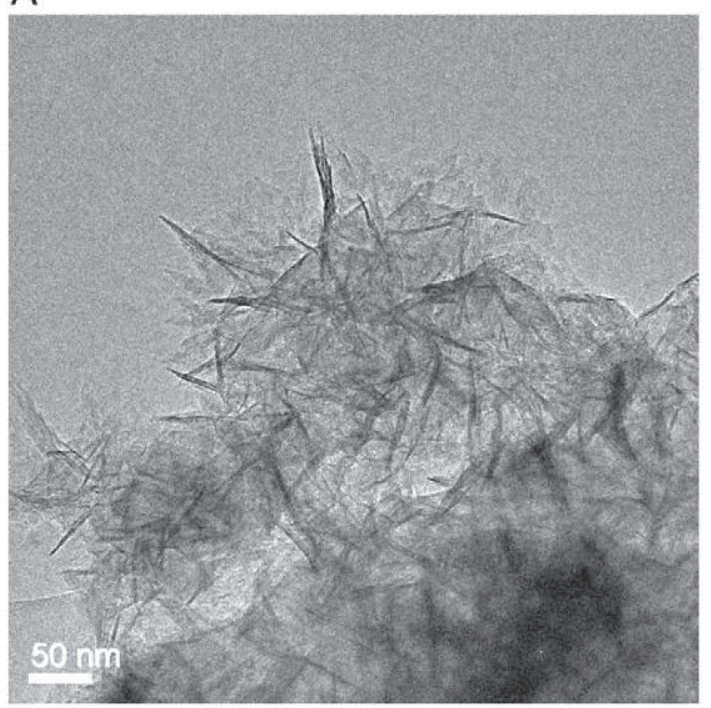

B

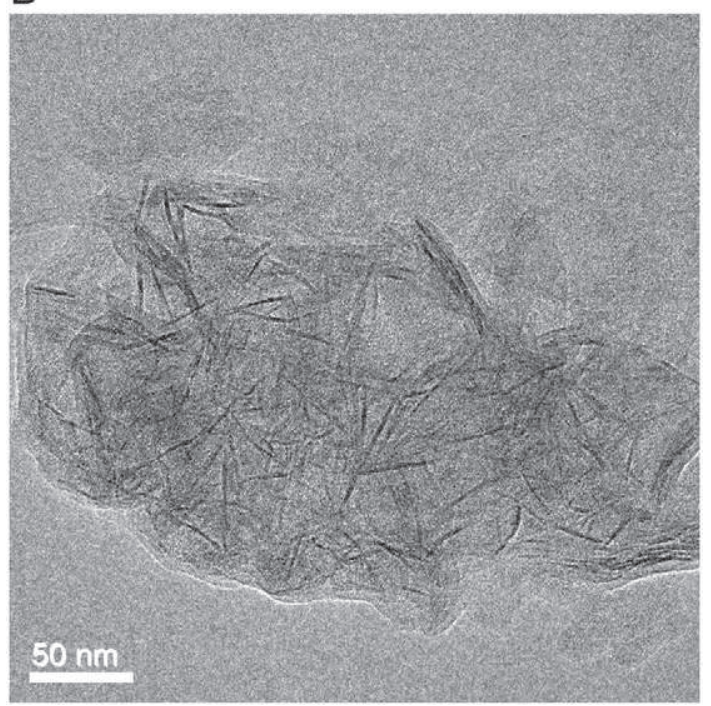

Fig. 4. TEM micrographs of (A) LP and (B) LP-P boehmite samples.

\section{Discussion}

\subsection{Size and shape of boehmite crystallites}

The thickness $\varepsilon_{020}$, along the b axis, calculated by the Scherrer's equation, is similar for all samples (Table 3 ). This means that the three synthesized boehmites have crystallites of equivalent sizes. The XRD simulation results show that, for all samples, the crystallites are diamond-shaped exhibiting essentially (010) and (101) faces with a thickness of $3 \pm 0.5 \mathrm{~nm}$. Moreover, for each family of samples, XRD patterns after peptization, neutralization and kneading-extrusion, are the same indicating that the shape and size of crystallites are not modified whatever the applied treatment. There is probably neither dissolution nor growth of boehmite particles during shaping process, especially during the peptization step. This result is consistent with the stability of the specific surface area whatever the applied step.

The special case of HP boehmite, showing a diffraction line at $2 \theta \approx 3.5^{\circ}$, indicates that this material present well organized crystallites. Although it cannot be unambiguously demonstrated from the XRD patterns, indeed, we think that the observed fibers are agglomerated diamond shaped particles rather than monocrystals. Indeed,
A

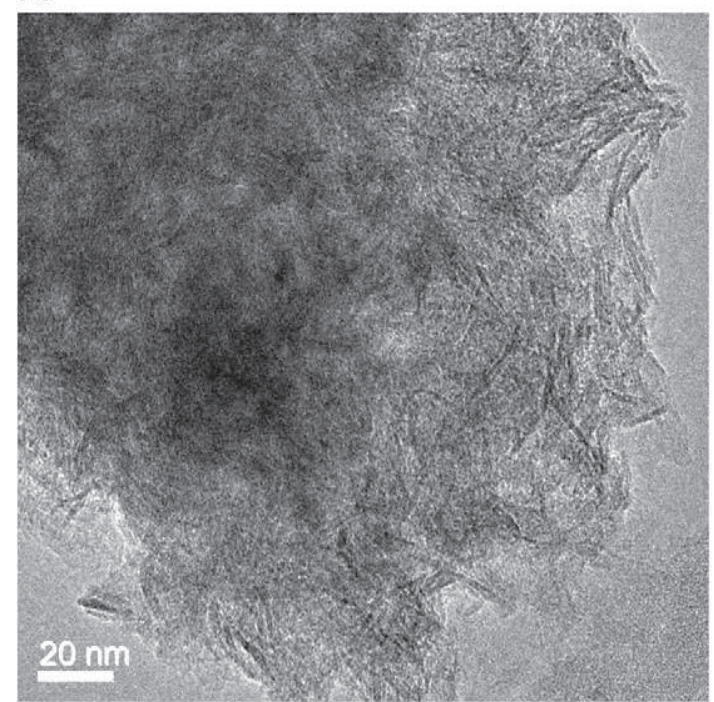

B

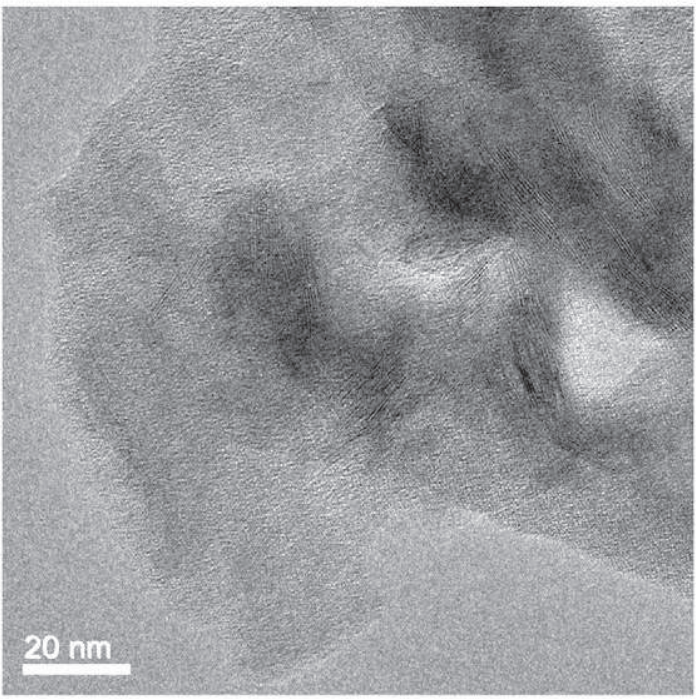

C

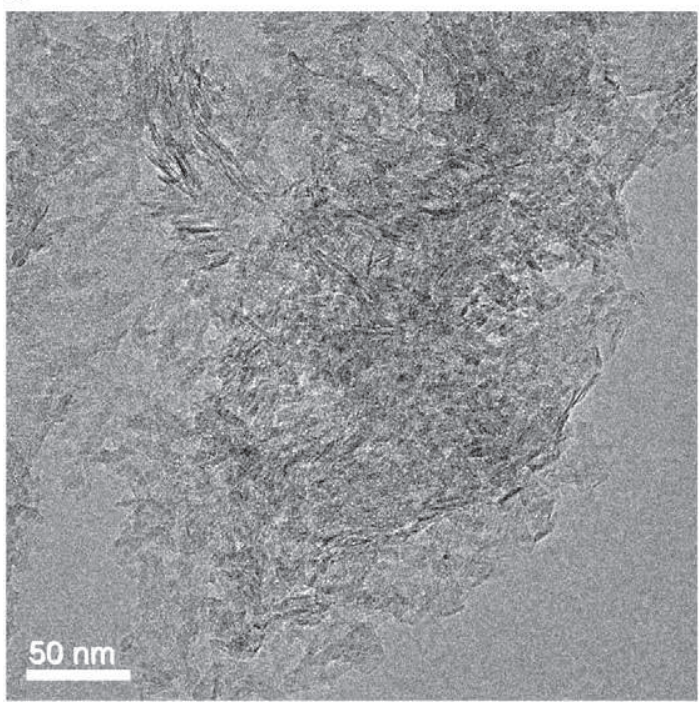

Fig. 5. TEM micrographs of boehmite samples (A) HP, (B) HP-P and (C) HP-PN. 

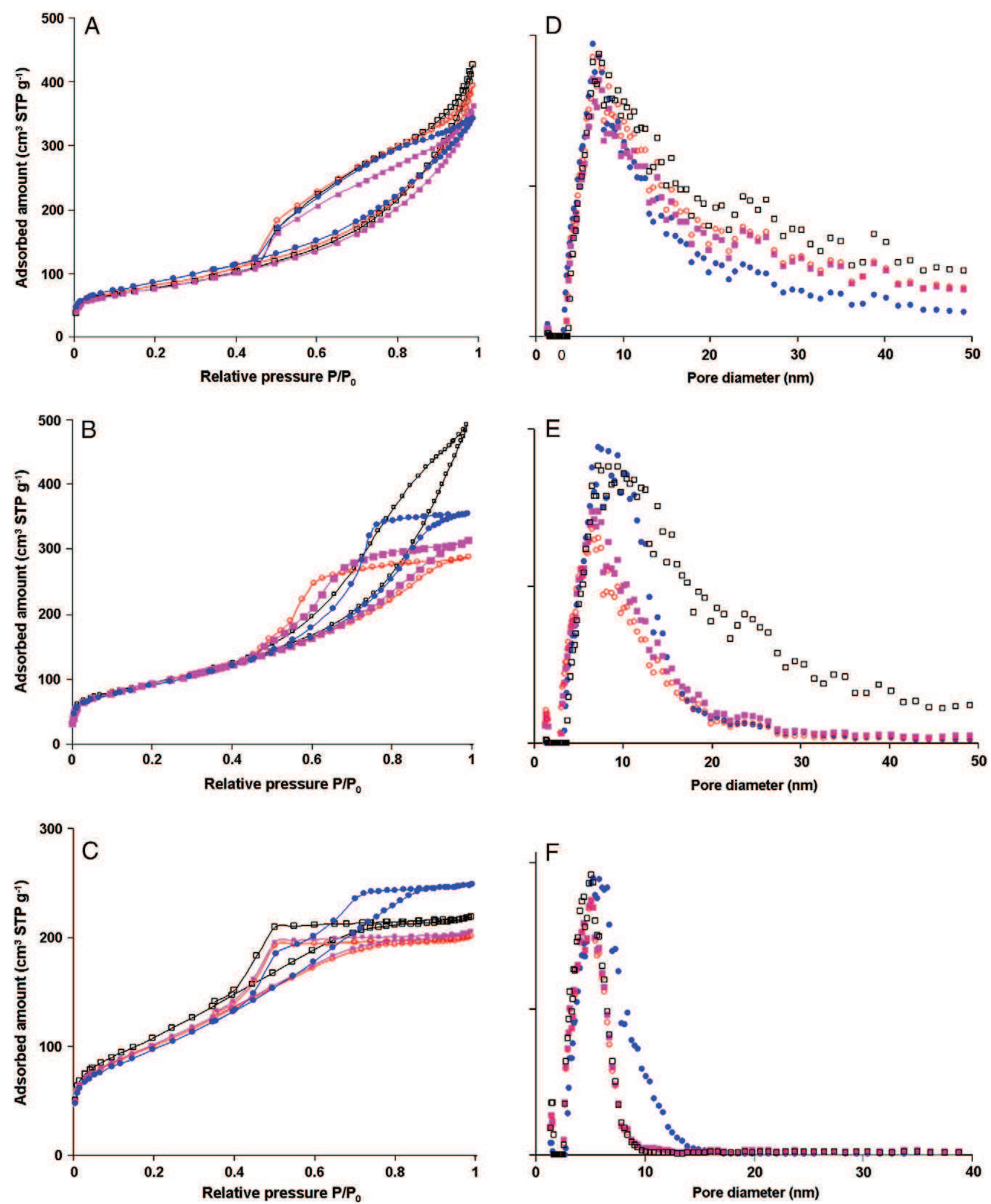

Fig. 6. Nitrogen adsorption-desorption isotherms, recorded at $77 \mathrm{~K}$, for as synthesized boehmites ( $\square$ ), peptized samples (O), neutralized samples ( $\square$ ), extrudates ( $\bullet$ ), and the corresponding pore size distribution calculated using the NLDFT method $(A \& D=L P, B \& E=M P$ and $C \& F=H P)$.

Mange et al. [27] have already demonstrated such behaviour for sol-gel derived boehmite where crystallites are organised in a highly oriented domain in the $(020)$ direction. Similar results have also been reported by Fukasawa et al. [28] where stacked layers of primary particles in the direction of the b-axis were observed for frozen or freeze-dried gels obtained by hydrolysis of aluminum isopropoxide.

TEM micrographs of the peptized HP sample corroborate this finding: initially observed fibrils are broken to form a homogeneous porous 
matrix when adding nitric acid. However, the shape and size of single crystallites could not be readily determined from TEM micrograph because of their nanometric size and the important aggregation state. These TEM micrographs are in agreement with the fact that HP boehmite is fully peptizable ( $\mathrm{PI}=0.99$ ). Nitric acid allows breaking down agglomerates to a size small enough to keep them in suspension. Conversely, for the LP boehmite, observed fibrils are mainly constituted by aggregates as no size reduction was observed after addition of nitric acid. This is in good agreement with the results of Morgado et al. [14] who shown that precipitation at high temperature did not favour the peptization. But contrary to Morgado et al. work [14] ${ }^{27} \mathrm{Al}$ MAS NMR (supporting information) did not reveal the presence of tetrahedral aluminum in none of the three boehmites, excluding the presence of amorphous boehmite that could impact the peptization behaviour. The partially peptizable MP boehmite is a mixture between agglomerates and aggregates small enough to remain in suspension (peptizable part) and larger agglomerates and aggregates which sediment (non peptizable part).

\subsection{Evolution of the textural properties}

Although LP and HP boehmites show an opposite behaviour regarding peptization, no significant changes are observed for the pore size distribution during the shaping process. For LP boehmite, the wide pore size distribution is due to a heterogeneous organisation of crystallites and aggregates generating voids ranging from $c a .5$ to $50 \mathrm{~nm}$ and the mean pore diameter is around $7.5 \mathrm{~nm}$. For shaped samples, no changes were observed, neither for the pore size distribution nor for the mean pore diameter, whatever the shaping process (step process or kneading-extrusion). These results can be explained by the fact that $\mathrm{LP}$ boehmite is non peptizable $(\mathrm{PI}=0)$. Hence, for non peptizable boehmites, no control of textural properties seems to be possible.

MP boehmite have more or less the same pore size distribution as LP but the mean pore diameter is around $9.5 \mathrm{~nm}$. This is due to the fact that both materials were obtained by precipitation, using the same precursors in equivalent conditions. However, the PSD of the
MP sample was strongly affected by peptization, neutralization and by the kneading-extrusion process. The as precipitated MP has a wide pore size distribution probably due to a heterogeneous organisation of aggregates and agglomerates. But the peptized and neutralized samples, respectively MP-P and MP-PN, gave narrower PSD than MP. During the peptization step, protons or hydroxyl ions adsorb on the crystallites surface causing electrostatic repulsion. This allows the particles to pack more efficiently while drying. This is why the pore size distribution narrowed (ranging from 5 to $20 \mathrm{~nm}$ ) and the mean pore diameter decreased to $6.5 \mathrm{~nm}$. When neutralization was carried out, a second pore size distribution appeared at $\approx 9.5 \mathrm{~nm}$ because neutralization of the surface charges caused particles agglomeration. However the range of the pore size distribution remained more or less the same. We also observe that for MP extrudates, the mean pore diameter was larger than the neutralized sample. This point is probably due to the kneader used for the kneading extrusion shaping process that would not allow the destruction of all agglomerates, probably because the knead was not enough homogeneous or the kneading not efficient enough to break down all aggregates.

HP boehmite has a narrower pore size distribution than LP or MP. The mean pore diameter around $5.1 \mathrm{~nm}$ for HP corresponds to the space between primary particles or small aggregates containing few crystallites. As observed in TEM micrographs, this sample is fully peptizable $(P I=0.99)$, nevertheless no significant changes in the pore size distributions were observed after peptization and neutralization steps. Thus, we assume that crystallites organised themselves in a compact configuration after the synthesis and that this spatial arrangement was retrieved after the peptization and the neutralisation steps. But two points have to be outlined: (i) this boehmite is fully peptizable, thus the crystallite organisation should be modified by peptization or neutralization and this was indeed observed on TEM micrographs; (ii) for the extrudates, the PSD is slightly modified and a shoulder is observed at $c a .8 .5 \mathrm{~nm}$. The first point seems to indicate that crystallites of peptized and neutralized samples using the Ultraturax ${ }^{\circledR}$ disperser tend to return to a spatial organisation

\section{As synthesised powder}
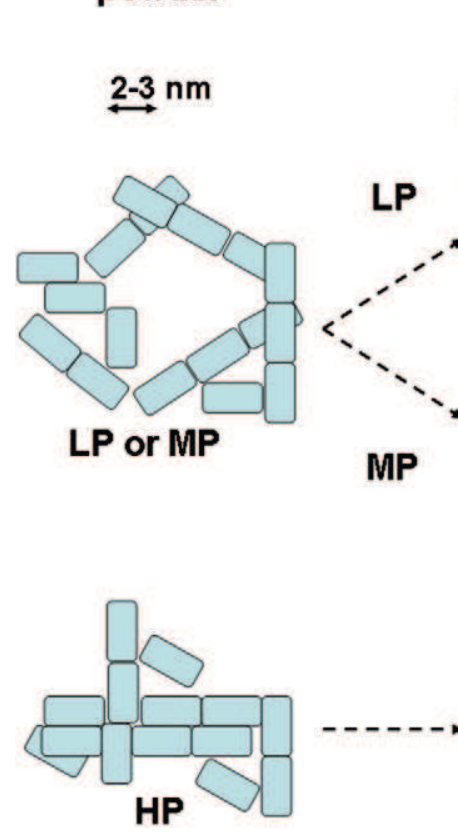

\section{After peptization}
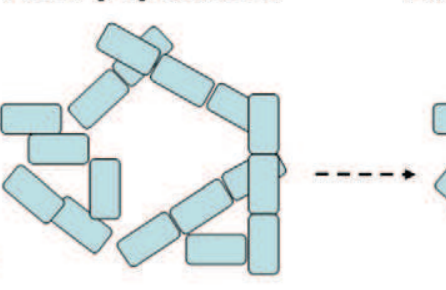

\section{After neutralization}

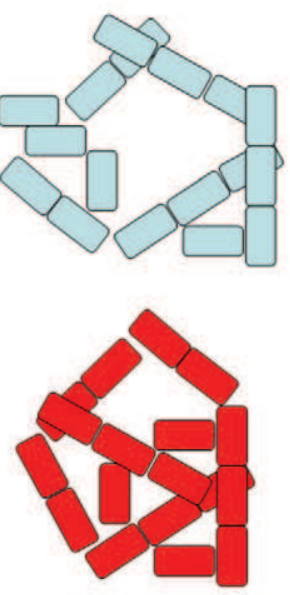

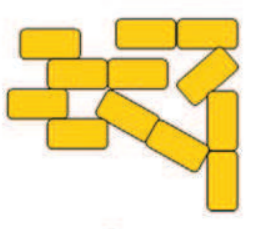
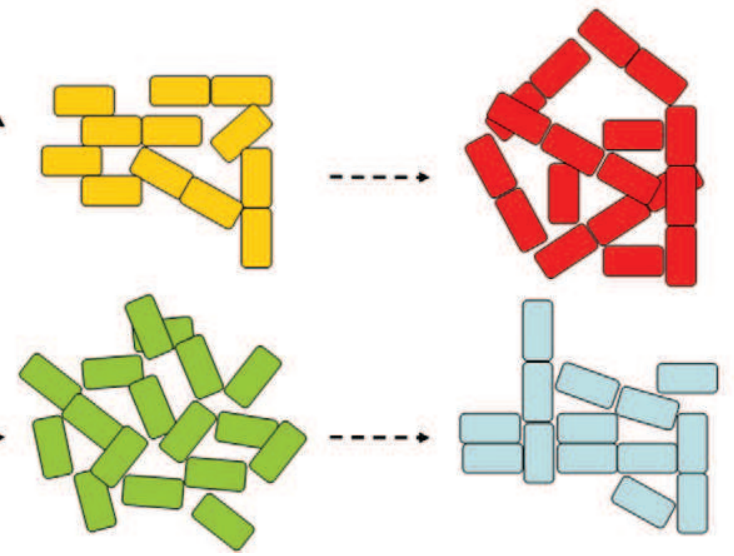

Fig. 7. Schematic illustration of boehmite nanoparticles assembly for as synthesized samples and their evolution during the shaping process. Different colors between two successive steps indicate significant changes of the particles assembly and as a consequence of the textural properties. 
close of the as synthesized material. Compared to the Brabender ${ }^{\circledR}$ mixer, one should keep in mind that using the Ultraturax ${ }^{\circledR}$ induces two important differences; (i) the mechanic work is different, (ii) the water/solid ratio $\approx 7$ is higher than the one $(0.5)$ used for the kneading-extrusion process. This could impact the textural properties of the obtained solids. The second one tends to confirm that a best knead was achieved while using the Ultraturax ${ }^{\circledR}$ disperser as it was observed for MP sample. In fact, the analysed paste kneaded using the Brabender ${ }^{\circledR}$ mixer has the same textural properties (specific surface area, pore volume and pore size distribution) as the extrudates. Thus it means that the extrusion process do not impact the textural properties of the past (see supporting information).

In order to control textural properties, the starting boehmite should not be poorly or highly peptizable but should have medium peptization ability. Fig. 7 summarizes the behaviour of boehmite crystallites as a function of the peptization ability of the starting material. For LP no changes in particle assembly were observed thus the textural properties were not modified. However, the particles organisation was modified for both MP and HP samples, the control of the textural properties were achievable only for MP sample: (i) as MP is partially peptizable, this allows to maintain some disorder in particles assembly that permit to tailor the textural properties, (ii) for HP, full dispersion of particles was achieved but the space between particles is equivalent to the one developed by as synthesized sample. After neutralisation, the particles return to a highly organized state so that no significant changes were observed in textural properties.

\section{Conclusion}

The textural properties (mainly, surface area, pore size distribution and pore volume) are functions of the size, shape and aggregation state of primary particles. But these properties can be tuned during the shaping process. For example, to tighten the pore size distribution, a reduction of the agglomerates size is used. This process, known as peptization, depends on the synthesis way and conditions. In this work, three boehmite materials with peptization indexes ranging from $c a$. 0 to $100 \%$ were prepared. The low peptizable boehmite (LP) was prepared by aluminum sulphate neutralisation at $60{ }^{\circ} \mathrm{C}$. The partially peptizable boehmite (MP) is an industrial product prepared by the same method that LP. The fully peptizable boehmite (HP) was obtained by hydrolysis of aluminum tri-sec-butoxide. Crystallites of both, not peptizable (LP) and fully peptizable (HP) boehmite agglomerate into fibrils. These fibrils are easily broken by nitric acid into a colloidal dispersion for HP, whereas no deagglomeration was observed for non peptizable boehmite (LP) because crystallites are strongly interconnected, forming a crystalline frame which could not be disrupted by nitric acid. Thus for a non peptizable boehmite, porosity seems to be more or less fixed by particle shape, size and aggregation state. Even if HP sample is highly peptizable, no significant changes are observed in the pore size distribution. Hence no tuning of textural properties appears to be possible in absence of additives. The more versatile sample to tune textural properties, especially the pore size distribution, is the partially peptizable MP sample.

\section{Acknowledgements}

The authors would like to thank the Physics and Analysis Division (IFPEN) for technical assistance.

\section{References}

[1] H.L. Zubowa, K. Baker, H.P. Henning U. Kretzschmar, M. Noack, U. Steinike, Possibilities of controlled synthesis of $\gamma-\mathrm{Al}_{2} \mathrm{O}_{3}$ pellets with a predetermined pore structure, Chemische Technik 41 (1989) 387-391.

[2] K. Strenge, U. Bollmann, A rheological investigation of peptized boehmite suspensions, Colloids and surfaces 57 (1991) 139-148.

[3] D.L. Trimm, A. Stanislaus, The control of pore-size in alumina catalyst supports, Applied Catalysis 21 (1986) 215-238.

[4] P. Euzen, P. Raybaud, X. Krokidis, H. Toulhoat, J.-P. Jolivet, C. Froidefond, Alumina, Handbook of Porous Solids, 2002, pp. 1591-1677.

[5] R. Bleta, P. Alphonse, L. Pin, M. Gressier, M.-J. Menu, An efficient route to aqueous phase synthesis of nanocrystalline $\gamma-\mathrm{Al}_{2} \mathrm{O}_{3}$ with high porosity: from stable boehmite colloids to large pore mesoporous alumina, Journal of Colloid and Interface Science 367 (2012) 120-128

[6] Stacy M. Grant, M. Jaroniec, Effect of cosolvent organic molecules on the adsorption and structural properties of soft-templated ordered mesoporous alumina, Journal of Colloid and Interface Science 367 (2012) 129-134.

[7] J. Nair, P. Nair, J.G. Van Ommen, J.R.H. Ross, A.J. Burggraaf, F. Mizukami, Influence of peptization and ethanol washing on the pore-structure evolution of sol-gel-derived alumina catalyst supports, Journal of the American Ceramic Society 81 (1998) 2709-2712.

[8] R. Brace, E. Matijevic, Aluminium hydrous oxide sols I: Spherical particles of narrow size distribution, Journal of Inorganic and NuclearChemestry 35 (1973) 3691-3705.

[9] D.L. Carone, E. Matijevic, Aluminium hydrous oxide sols II: preparation of uniform spherical particles by hydrolysis of Al sec-butoxide, Journal of Colloid and Interface Science 48 (1973) 291-301.

[10] W.B. Scott, E. Matijevic, Aluminium hydrous oxide sols III: preparation of uniform spherical particles by hydrolysis of aluminium chloride and perchlorate salts, Journal of Colloid and Interface Science 66 (1978) 447-454.

[11] D. Panias, A. Krestou, Effect of synthesis parameters on precipitation of nanocrystalline boehmite from aluminate solutions, Powder Technology 175 (2007) 163-173.

[12] D. Vorkapic, T. Matsoukas, Reversible agglomeration: a kinetic model for the peptization of titania nanocolloids, Journal of Colloid and Interface Science 214 (1999) 283-291.

[13] R. Petrovic, S. Milonjic, V. Jokanovic, L.J. Kostic-Gvozdenovic, I. Petrovic-Prelevic, D.J. Janackovic, Influence of synthesis parameters on the structure of boehmite sol particles, Powder Technology 133 (2003) 185-189.

[14] Edisson Morgado Jr., Y.L. Lam, L.F. Nazar, Formation of peptizable boehmites by hydrolysis of aluminium nitrate aqueous solution, Journal of Colloid and Interface Science 188 (1997) 257-269.

[15] J. Hille, U. Bollmann, W. Weinhold, H. Spindler, Preparation and properties of aluminium hydroxide III. Peptization of boehmite with nitric acid, Zeitschrift für Anorganische und Allgemeine Chemie 580 (1990) 188-198.

[16] R.K. Oberlander and L.B. Decker, Alumina dispersion behaviour, US patent 4360449 , (1982).

[17] V.D. Brink, Method of manufacturing a catalyst, US patent 7582588, (2009).

[18] J.F. Le Page, Catalyst forming, Preparation of Solid Catalysts, Federal Republic of Germany, Weinheim, 1999, pp. 579-589.

[19] Krijn P. de Jong, Synthesis of Solid Catalysts, Federal Republic of Germany, Weinheim, 2009.

[20] M.G. Sanchez, Extruded alumina catalyst support having controlled distribution of pore sizes, US patent 4301037, (1981).

[21] B.E. Yoldas, Transparent activated nonparticulate alumina and method of preparing same, US patent 3941719, (1976).

[22] S. Desset, O. Spalla, B. Cabane, Redispersion of alumina particles in water, Langmuir 16 (2000) 10495-10508.

[23] D. Chiche, M. Digne, R. Revel, C. Chaneac, J.P. Jolivet, Accurate determination of oxide nanoparticle size and shape based on X-ray powder pattern simulation: application to boehmite AlOOH, Journal of Physical Chemistry C 112 (2008) 8524-8533.

[24] D. Espinat, F. Thevenot, J. Grimoud, K. El Malki, Powerful new software for the simulation of WAXS ans SAXS diagrams, Journal of Applied Crystallography 26 (1992) 368-383.

[25] S. Brunouer, P.H. Emmet, E. Teller, Adsorption of gases in multimolecular layers, Journal of the American Chemical Society 60 (1938) 309-319.

[26] R. Evans, U.M.B. Marconi, P. Tarazona, Capillary condensation and adsorption in cylindrical and slit-like pores, Journal of the Chemical Society, Faraday Transactions 2: Molecular and Chemical Physics 82 (1986) 1763-1787.

[27] F. Mange, D. Fauchadour, L. Barré, L Normand, L Rouleau, A microstructural investigation of nanostructured boehmite films prepared by the sol-gel route, Colloids and Surfaces 155 (1999) 199-210.

[28] J.-I. Fukasawa, K. Tsujii, Higher-order structure formation of ultrafine boehmite particles in sols, gels, and dried materials, Journal of Colloid and Interface Science 125 (1987) 155-161. 